\title{
Coronary Artery Bypass Graft in a Young Adult, An Emerging Phenomenon: A Case Report.
}

\author{
Ramzisham ARMa , Joanna OSMc, Talal AR ${ }^{\mathrm{a}}$, Shahrulakmal $\mathrm{S}^{\mathrm{a}}$, Maskon $\mathrm{O}^{\mathrm{b}}$, Noorfaizan $\mathrm{S}^{\mathrm{b}}$ and Zamrin $\mathrm{DM}^{\mathrm{a}}$ \\ ${ }^{a}$ Division of Cardiothoracic Surgery, Department of Surgery \\ ${ }^{b}$ Division of Cardiology, Department of Medicine \\ ' Division of Cardiothoracic Anaesthesia, Department of Anaesthesiology and Intensive Care Heart and Lung \\ Centre, Hospital Universiti Kebangsaan Malaysia, Jalan Yaacob Latif, 56000 Cheras, Kuala Lumpur.
}

\begin{abstract}
A 29-year-old, smoker, diabetic, hypertensive and hypercholesterolemic man was diagnosed of having severe coronary artery disease with a critical left main stem stenosis. On-pump coronary artery bypass graft surgery (CABG) was performed using conduits from the left internal mammary artery as well as the left radial artery and right saphenous vein which were both harvested endoscopically. He was discharged after a week of uneventful recovery. This case emphasizes on the best possible management of conduits selection, intraoperative myocardial protection and the risk factors modification in achieving a long term graft patency and avoiding premature cardiac related death.
\end{abstract}

KEYWORDS: CABG, Myocardial infarction, Young

\section{INTRODUCTION}

Atherosclerotic coronary artery disease (CAD) is classically a known phenomenon in an elderly population. ${ }^{1-3}$ Recently, there have been an emergent of cases of young patients presented with acute coronary syndrome requiring intervention. ${ }^{2}$ The Framingham study reported a 10-year incidence rate per 1000 myocardial infarctions of 12.9 in men of 30 to 34 years old and 5.2 in women of 35 to 44 years old. ${ }^{3}$ Review of a few studies, however, proved only approximately $4 \%$ of patients who had myocardial infarction (MI) was below 40 years of age. ${ }^{3}$ So far, there was no reported data on the rate of incidence of $\mathrm{MI}$ and the incidence of CABG in patients below 30 years of age. Clinical and epidemiological studies have been conducted in identifying the risk factors and surgical outcomes in this group of patients. ${ }^{1,2}$ Although premature atherosclerosis begins in childhood, only a small subgroup of patients requires coronary revascularization at a very young age. ${ }^{4}$ When the coronary artery bypass graft surgery (CABG) is indicated in young patients, the choice of the operative technique, conduits selection and post-operative risk factors modification should be carefully considered to prolong the graft patency and to avoid premature death. ${ }^{5,6}$ We presented a case of a very young patient with proven risk factors who required $C A B G$. It is reported here for its rarity and exemplary case in which the treatment given was supported by the literature and had resulted in good outcome.

Corresponding author;

Dr Mohd Ramzisham Abdul Rahman

Division of Cardiothoracic Surgery

Department of Surgery

Universiti Kebangsaan Malaysia Medical Centre

e-mail: ramzisham@hotmail.com

\section{CASE REPORT}

A 29-year-old male was diagnosed to have an acute inferolateral myocardial infarction. He was known to have insulin-dependent diabetes mellitus (IDDM) since the age of 14 years old. His other risk factors include hypertension, hypercholesterolemia and cigarette smoking. He had a strong family history of diabetes and heart disease. Clinical examination and basic blood investigations revealed no significant abnormalities. A transthoracic echocardiography revealed a good left ventricular function with an ejection fraction of $65 \%$. The coronary angiography revealed a $70 \%$ stenosis of the distal left main stem, $90 \%$ stenosis at the proximal left anterior descending artery (LAD), $90 \%$ mid circumflex (CX) stenosis and total occlusion of the distal right coronary artery.

He underwent an urgent CABG. A standard median sternotomy was performed whereby the left internal mammary artery was harvested. The left radial artery (Figure 1) and right long saphenous vein were harvested endoscopically. Cardiopulmonary bypass was established with the temperature allowed to drift down to $30^{\circ} \mathrm{C}$. The aorta was cross clamped and myocardial preservation was done using both antegrade and retrograde cold blood cardioplegia with surface cooling of the heart using ice slush. The saphenous vein was grafted to the right posterior descending artery, left radial artery to the first obtuse marginal branch and the left internal mammary to the LAD. The total bypass time was 97 minutes with the cross clamp time of 56 minutes. The patient came off the cardiopulmonary bypass without much difficulty, with minimal use of intravenous infusion of adrenaline. He was extubated four hours following surgery and was managed in cardiac intensive care unit for only one day. He had an uncomplicated recovery and was discharged on the seventh post-operatively day. The last follow up was eighteen months after the surgery and 
he was remarkably well.

\section{DISCUSSION}

Coronary artery disease (CAD) requiring CABG is common in the middle age or elderly population. The peculiarity of our patient is that he had CABG done at a very young age of 29 years old. Although the prevalence of coronary atherosclerosis in young adults is difficult to estimate, data on the risk of developing CAD at an older age based on risk factors present during childhood had been reported. ${ }^{1,2,4}$ Those risks factors describe in various studies are present in our patient such as strong family history, presence of diabetes mellitus, hypercholesterolaemia, hypertension and heavy chronic smoking..$^{1-4}$ In the current era, primary prevention includes identifying the genetic risk as well as optimizing the modifiable risk factors. This is vital to this group of young population before the target organ damage occurs. ${ }^{4}$ Antiplatelets and lipid lowering drugs have been well established in many trials in inhibiting the atherosclerotic process and slow down the progression of the disease, and this should be the standard practice in current management. ${ }^{7}$

There is no argument that CABG remains the best option for young patient with multivessel CAD when left main stem is involved. CABG has been shown to relieve symptoms and improve the left ventricular function. ${ }^{5}$ Our case illustrated that CABG was appropriate in such patient and in our patient it had resulted in good long term outcome.

Selecting appropriate conduits for long lasting graft patency is another important issue in this patient. Several studies have shown the superiority of internal mammary artery over saphenous vein grafts in maintaining patency and improving the 10-year survival rate. ${ }^{5,6,8}$ The use of other arterial grafts such as radial artery was proven to be superior to vein grafts for long term patency especially on the left coronary system. ${ }^{9,10}$ When significant risk factors for the sternal wound complication are present such as IDDM and chronic heavy smoking as the case above, we prefer not to use bilateral IMAs. Furthermore, we feared that the right IMA may be stretched when the distal anastomosis has to be grafted far down onto the PDA branch. The use of endoscopic technique in harvesting the radial and saphenous vein like in this patient will certainly reduce the ill cosmetic effect in a young patient and the potential diabetic wound infection (Figure 2).

Intra-operative use of blood cardioplegia has resulted in a reduction in peri-operative AST level giving the best myocardial protection as proven by several studies. ${ }^{1,5}$ A combination of good myocardial protection and a bypass time of less than 100 minutes have been proven to reduce mortality and recurrence of post-operative ischaemic symptoms. ${ }^{5}$

\section{CONCLUSION}

In the current era, we must be aware of the increasing number of cases of very young patients with significant CAD. When surgical revascularization is considered, the patient must be given the best possible options in terms of the conduits and intra-operative myocardial protection in order to prolong the graft patency. Post-operative use of anti-platelet and lipid lowering drugs as well as modification of the risk factors are equally important to avoid premature cardiac related death. A close monitoring and a long term follow-up is mandatory in this group of patients.

\section{REFERENCES}

1. Mukherjee D, Hsu A, Moliterno DJ, Lincoff AM, Goormastic M, Topol EJ. Risk factors for premature coronary artery disease and determinants of adverse outcomes after revascularization in patients 40 years old. Am J Cardiol 2003; 92:1465-7

2. Kanitz MG, Giovannucci SJ, Jones JS, Mott M. Myocardial infarction in young adults: Risk factors and clinical features. J Emerg Med 1996; 14:13945

3. Zimmerman FH, Cameron A, Fisher LD, Grace NG. Myocardial infarction in young adults: Angiographic characterization, risk factors and prognosis. JACC 1995; 26: 654-61

4. McGill HC, MCMahan CA. Determinants of artherosclerosis in the young. Pathobiological determinants of atherosclerosis in youth research group. Am J Cardiol 1998; 82:30T-36T

5. Ng WK, Vedder M, Whitlock RML, et al. Coronary revascularization in young adults. Eur $\mathrm{J}$ Cardiothor Surg 1997; 11:732-8

6. Abdul-Rahman MR, Ghaie S, Sadaba JR, et al. A survey of control of major risk factors following coronary artery bypass graft surgery. $\mathrm{Br} \mathrm{J}$ Cardiol 2002; 9:153-7

7. Goyal A, Alexander JH, Hafley GE, et al. Outcomes associated with the use of secondary prevention medications after coronary artery bypass graft surgery. Ann Thorac Surg 2007; 83:993-1001

8. Cole JH, Miller JI, Sperling LS, Weintraub WS. Long term follow-up of coronary artery disease presenting in young adults. J Am Coll Cardiol 2003; 41:521-8

9. Malinowski M, Mrozek R, Twardowski R, et al. Left internal mammary artery improves 5 -year survival in patients under 40 subjected to surgical revascularization. Heart Surg Forum 2006; 9:E493-7

10.Zacharias A, Habib RH, Schwann TA, et al. Radial artery conduits in coronary artery bypass grafting: Current perspective. J Thorac Cardiovasc Surg 2005; 130:232-33 


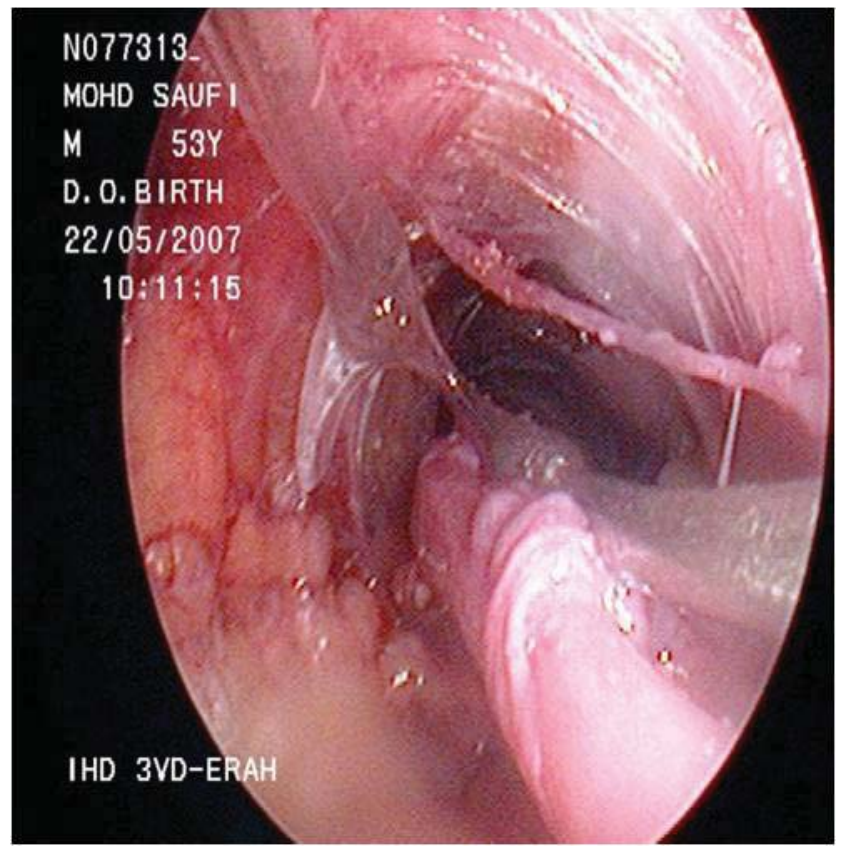

Figure 1. Endoscopic radial artery harvest

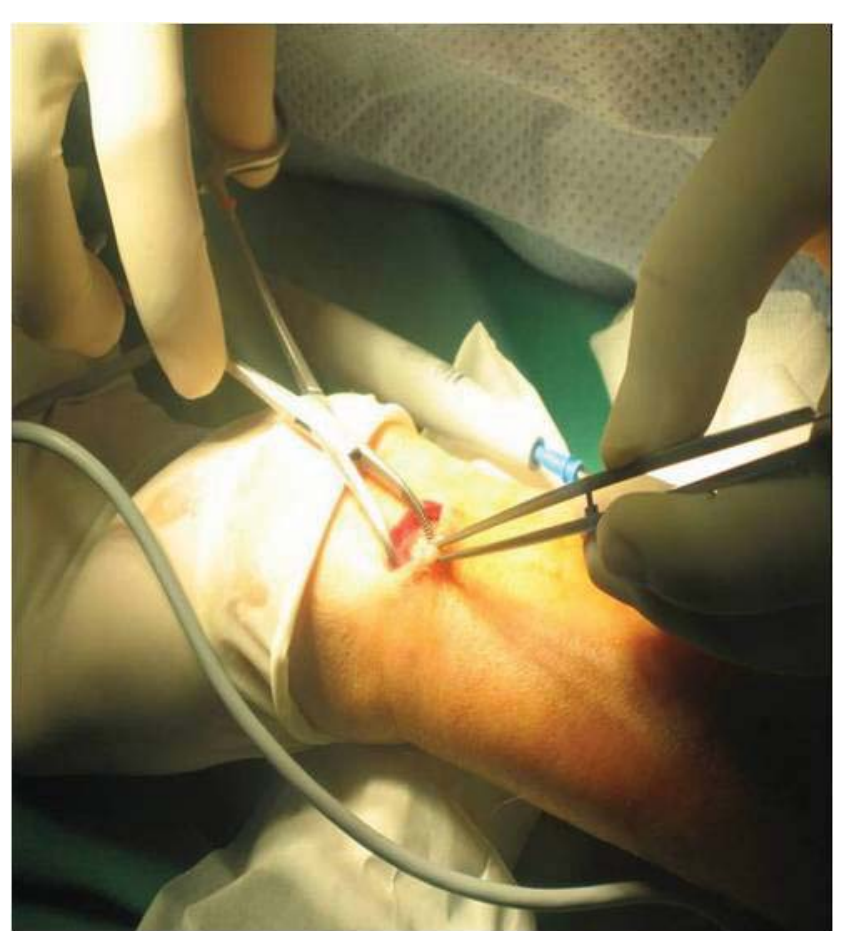

Figure 2. Small wound following endoscopic radial artery harvest 
